\title{
Effectiveness of a Short Duration Training Programme in the Physical Fitness of Patients with High-Moderate Risk of Cardiovascular Disease
}

\author{
Felícitas García-Ortún ${ }^{1 *}$, Angeles Jaén², Laura Sucarrats3 ${ }^{3}$, Lidia González-Gile, \\ Sandra Sabarich ${ }^{5}$, Judit Novo ${ }^{5}$, Alejandro de la Sierra', Amparo Alvarez-Auñón ${ }^{7}$, \\ Roser Garreta ${ }^{1,3}$ \\ ${ }^{1}$ Cardio-Respiratory Unit of Rehabilitation Service of Hospital Universitari Mútua Terrassa, Terrassa, Spain \\ ${ }^{2}$ Research Unit of Fundació Docència i Recerca Mútua Terrassa, Terrassa, Spain \\ ${ }^{3}$ Rehabilitation and Biomechanics Services, Mutual Insurance Company for Occupational Accidentes and \\ Diseases, Egarsat, Spain \\ ${ }^{4}$ Primary Care Rambla Centre of Hospital Universitari Mútua Terrassa, Terrassa, Spain \\ ${ }^{5}$ Primary Care Sud Centre of Hospital Universitari Mútua Terrassa, Terrassa, Spain \\ ${ }^{6}$ Internal Medicine Service of Hospital Universitari Mútua Terrassa, Terrassa, Spain \\ ${ }^{7}$ Cardiology Service of Hospital Universitari Mútua Terrassa, Terrassa, Spain \\ Email: ${ }^{\text {feligaror@hotmail.com }}$
}

Received 27 February 2015; accepted 25 May 2015; published 29 May 2015

Copyright (C) 2015 by authors and Scientific Research Publishing Inc.

This work is licensed under the Creative Commons Attribution International License (CC BY).

http://creativecommons.org/licenses/by/4.0/

(c) (i) Open Access

\section{Abstract}

Introduction: It is not known what the minimum duration of training programme is effective in primary prevention of cardiovascular disease. The objective of this study is to determine the effectiveness in physical fitness and exercise of the training programme of 8 weeks of duration with two modalities ( 2 or 3 sessions per week) in subjects with moderate-high risk of cardiovascular disease in a primary care setting. Main Findings: A significant increase in the six-minute walk test was observed (from $488 \pm 87.4 \mathrm{~m}$ to $532 \pm 68.0 \mathrm{~m}, \mathrm{p}<0.0001$ ). A significant increase of exercise duration (from $11.4 \pm 3.0 \mathrm{~min}$ to $12.3 \pm 3.0 \mathrm{~min}, \mathrm{p}<0.0001$ ) and a delay in the anaerobic threshold (from $8.8 \pm 2.9 \mathrm{~min}$ to $10.1 \pm 2.5 \mathrm{~min}, \mathrm{p}<0.0001$ ) during the ergometric tests were also observed. The proportion of patients exercising regularly increased significantly (from $36.1 \%$ to $83.3 \%, p<0.0001$ ). Both modalities of training programme improved fitness and physical activity, without significant differences between them. Conclusion: Our study shows that

*Corresponding author.

How to cite this paper: García-Ortún, F., et al. (2015) Effectiveness of a Short Duration Training Programme in the Physical Fitness of Patients with High-Moderate Risk of Cardiovascular Disease. Open Journal of Preventive Medicine, 5, 218-226. 
a structured training programme of two months of duration with 2 or 3 sessions per week significantly improves the fitness and physical activity of patients with moderate-high risk of cardiovascular disease.

\section{Keywords}

Cardiovascular Disease, Cardiovascular Risk Factor, Physical Exercise

\section{Introduction}

The primary prevention of cardiovascular disease (CVD) is a significant public health issue that remains unsolved.

A sedentary style of life and low cardiorespiratory fitness increase the risk of CVD, and their impact is similar to that of smoking, hypertension or dyslipidemia [1]. Physical fitness is an independent predictor of morbidity and mortality [2]. In the European Union, $62.4 \%$ of the population older than 15 years of age have a sedentary style of life. The percentage of sedentarism reaches $71 \%$ in Spain and Germany, only surpassed by that of Belgium and Portugal [3].

Several interventions have been used to improve physical fitness and enhance life style. Structured training programmes (TP) have been used to improve fitness and other CVD risk factors in the short-medium term [1] [4]-[8]. However, it is not known what is the minimum programme duration required to obtain significant results. Most studies conducted on CVD primary prevention have investigated TP with a duration longer than 12 weeks [1] [4]-[7], [9]-[13]. The few studies investigating the effectiveness of short duration TP $(\leq 8$ weeks) were conducted outside primary care, mainly on diabetic patients, with small sample sizes and inconclusive results [14]-[17]. It seems that the TP are effective when they have duration of more than 3 months. However, the effectiveness is unknown in training programs of shorter duration. Due to the economic situation of the country and the need to optimize resources, our group started a study with the aim of determine the effectiveness on improvement in physical fitness and physical activity of the training program of 8 weeks duration.

\section{Materials and Methods}

\subsection{Design Overview}

This is a prevention intervention with before-after design and comparative study between groups with different frequency sessions.

\subsection{Setting and Participants}

Patients with high-moderate risk of CVD were consecutively and prospectively recruited for January 2011 to December 2012 in two primary care centres in the reference area of Hospital Universitari Mútua Terrassa. Inclusion criteria: Patients of 35 - 74 years of age, with high-moderate risk of CVD, but free of overt disease. The high-moderate risk of CVD was considered on the presence of type 2 diabetes mellitus or metabolic syndrome (according to the National Cholesterol Education Program/American Heart Association criteria [18] or hypertension with at least one associated CVD risk factors (smoking, dyslipidemia, or obesity). Presence of hypertension or dyslipidemia were diagnosed if the patient was receiving specific pharmacological treatment for these conditions or if the following values were reached: systolic arterial blood pressure $>140 \mathrm{mmHg}$ or diastolic arterial blood pressure $>90 \mathrm{mmHg}$ in non-diabetic patients, and systolic arterial blood pressure $>130 \mathrm{mmHg}$ or diastolic arterial blood pressure $>80 \mathrm{mmHg}$ in diabetics, registered in 3 o more occasions; Total cholesterol levels $>190 \mathrm{mg} / \mathrm{dL}$ or Low Density Lipoprotein cholesterol levels $>115 \mathrm{mg} / \mathrm{dL}$ in non-diabetics and total cholesterol levels $>175 \mathrm{mg} / \mathrm{dL}$ or Low Density Lipoprotein cholesterol levels $>100 \mathrm{mg} / \mathrm{dL}$ in diabetics [19]. Diabetes mellitus were diagnosed if the patients was receiving specific pharmacological treatment or glycosylated haemoglobin levels $>6.5 \%$ or if fasting plasma glucose $>110 \mathrm{mg} / \mathrm{dL}$ [19]. Obesity were diagnosed if the body mass index 
$\geq 25$ [19]. Exclusion criteria: patients with CVD disease; last clinical appointment more than one year ago; pregnancy; disabling comorbidities. All patients gave informed consent to participate in the study. The study was approved by the Ethical Committee of Hospital Universitari Mutua Terrassa.

\subsection{Randomization and Intervention}

A total of 94 patients were recruited for the study of them 13 were excluded for lack of adherence to the programme. The final sample $(\mathrm{N}=81)$ were all of them evaluated at the end of the programme.

All included patients were assigned consecutively without randomization in two intervention groups depending on logistic and adherence reason related with patients. Both groups were performed a TP during two months, one of them twice a week (TP2) and the other three times a week (TP3). Of the total of 81 studied patients 32 performed TP2 and 49 TP3.

A structured TP was conducted for both groups (TP2 and TP3) in series of 6 participants each under the supervision of a physiotherapist. Each session lasted 60 minutes and consisted of: 5 minutes of warming up, 30 minutes of endurance training on a treadmill walking, cycle ergometer or on a cross trainer, 15 minutes of isotonic resistance (weight ) training of the large muscle groups of the upper and lower body, and 10 minutes of cool-down. The intensity of the endurance or resistance training was adjusted for each patient according to European Association for Cardiovascular Prevention \& Rehabilitation recommendation [20] taking into account their CVD risk factors and considering the anaerobic threshold (AT) level determined during the cardiopulmonary exercise testing (CPET) as the reference parameter. During the resistance training, two sets of 10 - 12 repetitions were performed at each station. The load was adjusted for each patient according to the muscular fatigue observed (5 - 6 out of 10 according to a visual analogue scale). In addition to the TP, two informative talks about CVD, healthy diet and exercise were offered.

As exercise test we performed 6 Minute walking test (6MWT) and CPET. Both tests were performed following the guidelines of the Spanish Society of Pneumology and Thoracic Surgery (SEPAR) [21] and of the European Respiratory Society [22]. The CPET by treadmill was performed following the modified protocol of Bruce, except in those cases with associated co-morbidity or lack of motor skills, where a cycle ergometer with workload increases of 25 watts every 2 minutes was used. Maximum CPET was considered when the respiratory quotient peak was more than 1.1 at the end of the CPET [23]. The AT was determined from the gas exchange analyses using the V-slope method [24]. In those cases where the V-slope was difficult to determine, the ventilatory equivalent method was used [25].

\subsection{Outcomes and Follow-Up}

The primary endpoint was the change in the result of the six-minute walk test (6MWT). Secondary endpoints included ergometric data (peak oxygen uptake $(\mathrm{ml} / \mathrm{kg} / \mathrm{min}$ ) (VO2p), exercise duration, AT expressed as VO2 and as time (minutes), double product (heart rate $\times$ systolic arterial blood pressure) basal and at peak of exercise), frequency and duration of exercise, anthropometric (weight, body mass index, abdominal perimeter), analytical (glucose, glycosylated haemoglobin, total cholesterol, Low Density Lipoprotein cholesterol levels and High Density Lipoprotein cholesterol levels, and triglycerides), systolic arterial blood pressure, diastolic arterial blood pressure and smoking habits. The waist circumference was measured from the point midway between the inferior margin of the last rib and the iliac crest, above the umbilicus in a relaxed standing position. Blood pressure was measured using a mercury sphygmomanometer after 5 minutes rest.

Regular exercise was considered when the patient exercised moderately or vigorously for at least 30 minutes 3 times a week.

All study variables were collected at basal point and after the completion of the TP.

\subsection{Sample Power Calculations}

The sample size required to determine the effectiveness of the TP was based on the 6MWT published results (difference $\geq 35$ meters) [26]. Twenty five individuals per group will give a power of $80 \%$ for a correlation coefficient before-after of 0.7. To determine differences between TP2 and TP3 (with alpha $=0.05$ and Beta $<0.2$, two-sided), 25 subjects per group were required to detect differences $\geq 35$ metres in the 6MWT, considering a standard deviation of 65 , a correlation between measurement of 0.8 , and an experimental loss of $10 \%$. 


\subsection{Statistical Analysis}

As the subjects were included in a group with 2 weekly training sessions (TP2) or a group with 3 weekly training sessions (TP2) without randomisation, we performed the comparative analyses among twenty-seven patients per group (TP2 and TP3), those matched by age and gender in order to minimise bias of design.

Comparisons before and after the interventions were performed with all samples, and within and between TP2 and TP3 matched groups. Non-parametric tests were performed to analyse the data. Wilcoxon signed-rank tests were used for the analyses of quantitative data and Mc Nemar exact tests for qualitative data. For comparisons between TP2 and TP3, Fisher exact test, covariance analysis or regression were used as appropriate, considering the basal values of the corresponding variable. A $\mathrm{p} \leq 0.05$ was considered statistically significant. The statistical packages Stata SE and SPSS v17 were used for the analyses.

\section{Results}

The final sample $(\mathrm{N}=81)$ were all of them evaluated at the end of the programme. The average age were $62.2 \pm$ 6.3 years and $71.6 \%$ were males. The most frequent CVD risk factors observed in the sample was diabetes mellitus (76.5), followed by hypertension (65.4\%), dyslipidemia (64.2\%) and smoking (27.5\%). As shown in Table 1 a significant increase in the walked distance during the 6MWT test was observed (from $488 \pm 87.4 \mathrm{~m}$ to $532 \pm$ $68.0 \mathrm{~m}, \mathrm{p}<0.0001$ ). The respiratory quotient peak was always lower than 1.1 in all EGA performed indicated a submaximal effort. A significant delay in the AT (from $8.8 \pm 2.9 \mathrm{~min}$ to $10.1 \pm 2.5 \mathrm{~min}, \mathrm{p}<0.0001$ ) and a significant increase in exercise duration (from $11.4 \pm 3.0 \mathrm{~min}$ to $12.3 \pm 3.0 \mathrm{~min}, \mathrm{p}<0.0001$ ) were observed. The basal and peak double product showed a decrease $(10,307 \pm 1909$ to $9820 \pm 2276$ and 30,679 $\pm 36,647$ to 25,317 \pm 4728 , respectively; $\mathrm{p}<0.05$ ). A $47.2 \%$ increase in exercise practice was observed ( $\mathrm{p}<0.0001$ ), with a significant increase in both, the number of weekly sessions and the duration of the sessions.

Table 2 summarises the anthropometric, analytical and blood pressure data pre and post intervention in the total sample. A significant reduction in systolic arterial blood pressure and diastolic arterial blood pressure was observed (from $144 \pm 19.9$ to $140 \pm 15.5$, p $=0.0277$ and from $82.5 \pm 8.8$ to $79.3 \pm 7.9, \mathrm{p}=0.0012$, respectively). No anthropometric or analytical changes were observed. Only one patient gave up smoking after the programme.

Table 3 summarises the results of comparisons between and within groups. Regarding to differences within groups (pre-post TP) we observed significant increases in the walked distance during the 6MWT (from $496 \pm$ $80.9 \mathrm{~m}$ to $540 \pm 73.7 \mathrm{~min} \mathrm{TP2}$; $<0.001$, and from $482 \pm 109.8 \mathrm{~m}$ to $521 \pm 59.4 \mathrm{~min}$ TP3; $\mathrm{p}<0.01$ ), in the duration of the exercise (from $11.2 \pm 3.3 \mathrm{~min}$ to $12.3 \pm 3.1 \mathrm{~min}$ in TP2; $\mathrm{p}<0.01$, and from $11.0 \pm 2.5 \mathrm{~min}$ to $11.8 \pm$ 2.9 min in TP3; $\mathrm{p}<0.05$ ). A delay in the AT was observed in both groups but only were significant in TP3 (from $9.0 \pm 3.3 \mathrm{~min}$ to $10.1 \pm 2.6 \mathrm{~min}$ in TP2; $\mathrm{p}=0.058$, and from $8.3 \pm 2.5 \mathrm{~min}$ to $9.7 \pm 1.9 \mathrm{~min}$ in $\mathrm{TP} 3$; $\mathrm{p}<$ $0.001)$.

We observed a significant increase of proportion of patients that referred regular exercise practice post TP in both groups (TP2: $40.0 \%$ to $84.0 \%$ and TP3: $32.0 \%$ to $80.0 \%$; $\mathrm{p}<0.001$ ). Additionally, the frequency and the duration of weekly exercise increased similarly in both groups (Table 3).

There were no significant differences between groups in any of the study variables (Table 3).

No side effects were observed during the CPET. A patient suffered from acute severe hypertension and another from angina during exercise sessions. Both patients recovered after cessation of exercise and pharmacological treatment.

\section{Discussion}

Our study shows that a two month structured TP, with 2 or 3 sessions per week, increases significantly the physical fitness and physical exercise of patients with moderate-high risk of CVD but without overt disease. This is the first study that analyse the effectiveness of a shorter duration TP on a primary care setting and in patients with a range of risk factors including diabetes, hypertension, dyslipidemia, smoking, and obesity.

The TP improved the fitness of patients, with a significant increase in the 6MWT of $43.7 \pm 61.5 \mathrm{~m}$. Several studies have been used this test to evaluate fitness changes in the context of TPs but not in primary care with our target population. There is only one previous study [26] in which this test was used to analyse the results of several educational interventions with similar characteristics of setting and target population as ours study. These authors reported a maximum increase in the 6MWT test of $35 \mathrm{~m}$ with the most intensive educational intervention. 
Table 1. Changes in physical fitness and exercise in the final sample $(\mathrm{n}=81)$.

\begin{tabular}{|c|c|c|c|}
\hline & Basal Mean (SD) & Final TP Mean (SD) & $\mathrm{p}$ \\
\hline \multirow[t]{2}{*}{ 6MWT, meters $(\mathrm{n}=73)^{*}$} & $488(87.4)$ & $532(68.0)$ & $\leq 0.001$ \\
\hline & Ergometric data & & \\
\hline $\mathrm{HR} \times \mathrm{SBP}$ basal $(\mathrm{n}=76)^{*}$ & $10,307(1,909)$ & $9820(2276)$ & 0.04 \\
\hline HR $\times$ SBP peak $(n=74)^{*}$ & $30,679(36,647)$ & 25,317 (4728) & 0.03 \\
\hline VO2p, $\mathrm{ml} / \mathrm{kg} /$ minute $(\mathrm{n}=54)^{*}$ & $22.8(5.3)$ & $22.7(5.6)$ & 0.98 \\
\hline Exercise duration, minutes $(\mathrm{n}=81)^{*}$ & $11.4(3.0)$ & $12.3(3.0)$ & $\leq 0.001$ \\
\hline VO2AT, $\mathrm{ml} / \mathrm{kg} /$ minute $(\mathrm{n}=48)^{*}$ & $16.9(3.6)$ & $16.7(4.1)$ & 0.63 \\
\hline AT, minutes $(n=50)^{*}$ & $8.8(2.9)$ & $10.1(2.5)$ & $\leq 0.001$ \\
\hline Physical Exercise, $n(\%)(n=72)^{*}$ & $26(36.1)$ & $60(83.3)$ & $\leq 0.001$ \\
\hline Frequency,days/week $(\mathrm{n}=30)^{*}$ & $5.4(1.9)$ & $6.2(1.6)$ & 0.01 \\
\hline Duration, minutes/session $(\mathrm{n}=31)^{*}$ & $52.3(20.5)$ & $67.3(21.5)$ & $\leq 0.001$ \\
\hline
\end{tabular}

AT, Anaerobic threshold ; HR, heart rate; VO2AT, Oxygen consumption in AT; VO2p Peak Oxygen Uptake; 6MWT, Six Minute Walking Test; SBP, systolic arterial blood pressure; TP, Training Programme. ${ }^{*}=$ Number of patients with pre-TP and post-TP data.

Table 2. Changes in anthropometric, analytical and blood pressure data in the final sample $(\mathrm{n}=81)$.

\begin{tabular}{|c|c|c|c|}
\hline & Basal TP Mean (SD) & Final TP Mean (SD) & $\mathrm{p}$ \\
\hline \multicolumn{4}{|c|}{ Anthropometric data } \\
\hline Weight, $\mathrm{kg}(\mathrm{n}=81)^{*}$ & $83.4(12.8)$ & $83.3(12.5)$ & 0.98 \\
\hline $\operatorname{BMI}(\mathrm{n}=81)^{*}$ & $30.6(4.3)$ & $30.6(4.2)$ & 0.99 \\
\hline Abdominal perimeter men, $\quad \mathrm{cm}(\mathrm{n}=54)^{*}$ & $107(10.1)$ & $106(9.7)$ & 0.71 \\
\hline Abdominal perimeter women, $\mathrm{cm}(\mathrm{n}=21)^{*}$ & $109(8.3)$ & $110(8.1)$ & 0.71 \\
\hline \multicolumn{4}{|c|}{ Analytical data } \\
\hline glucose, $\mathrm{mg} / \mathrm{dl}(\mathrm{n}=44)^{*}$ & $183(71.5)$ & $172(61.4)$ & 0.40 \\
\hline HbA1c, $\%(n=31)^{*}$ & $7.9(2.0)$ & $7.4(1.4)$ & 0.09 \\
\hline Total cholesterol, $\mathrm{mg} / \mathrm{dl}(\mathrm{n}=35)^{*}$ & 198 (39.9) & $187(34.9)$ & 0.32 \\
\hline c-HDL, mg/dl (n = 32) & $44.7(9.7)$ & $46.2(10.8)$ & 0.43 \\
\hline c-LDL, $\mathrm{mg} / \mathrm{dl}(\mathrm{n}=30)^{*}$ & $124(34.1)$ & $109(26.5)$ & 0.10 \\
\hline triglycerides, $\mathrm{mg} / \mathrm{dl}(\mathrm{n}=33)^{*}$ & $145(64.6)$ & $140(55.7)$ & 0.71 \\
\hline SBP, $\operatorname{mmHg}(n=81)^{*}$ & $144(19.9)$ & $140(15.5)$ & 0.03 \\
\hline DBP, $\mathrm{mmHg}(\mathrm{n}=81)^{*}$ & $82.5(8.8)$ & $79.3(7.9)$ & $\leq 0.001$ \\
\hline
\end{tabular}

BMI, Body Mass Index; DBP, Diastolic Blood Pressure; HbA1c,Glycosylated Haemoglobin; c-HDL, High Density Lipoprotein cholesterol; c-LDL, Low Density Lipoprotein cholesterol; SBP, Systolic Blood Pressure; TP, Training Programme; "Number of patients with pre-TP and post TP data.

A delay in the AT and an increase in the duration of the exercise were observed during the CPET conducted after the TP, although no significant changes were observed in the AT expressed as VO2 and VO2p. The use of short stage (2 minutes) in the ergometry protocols may have influenced the accuracy of the AT determinations, and may partially explain the lack of significant changes in the AT expressed as VO2 [27] [28]. The lack of improvement in the VO2p may be explained by insufficient effort (respiratory quotient peak < 1.1) and/or low intensity of the training. The study patients could not perform maximum efforts during the CPET given their lack of fitness and co-morbidities. In contrast, Boudou et al. [16] observed significant improvement in VO2p with an 
Table 3. Changes in physical fitness and physical exercise between and within groups.

\begin{tabular}{|c|c|c|c|c|c|}
\hline & TP2 basal $(n=27)$ & TP2 final $(\mathrm{n}=27)$ & TP3 basal $(\mathrm{n}=27)$ & TP3 final $(n=27)$ & $\mathrm{p}^{*}$ \\
\hline \multicolumn{6}{|c|}{ Exercise test, mean (SD) } \\
\hline 6MWT, meters & $496(80.9)$ & $540(73.7)^{\S}$ & 482(109.8) & $521(59.4)^{\ddagger}$ & 0.47 \\
\hline $\mathrm{HR} \times \mathrm{SBP}$ basal & $10,410.4(1,634.2)$ & $9,930.2(2,014.0)$ & $10,185.7(1,987.7)$ & $9,250.2(2,725.2)$ & 0.59 \\
\hline $\mathrm{HR} \times \mathrm{SBP}$ peak & $26,523.6(5,219.3)$ & $25,722.3(4,285.3)$ & $27,185.2(5,365.1)$ & $25,475(5,223.6)$ & 0.86 \\
\hline VO2p, $\mathrm{ml} / \mathrm{kg} /$ minute & $23.7(5.1)$ & $23.3(5.1)$ & $23.0(5.6)$ & $22.0(6.6)$ & 0.73 \\
\hline Exercise Duration, minutes & $11.2(3.3)$ & $12.3(3.1)^{\ddagger}$ & $11.0(2.5)$ & $11.8(2.9)^{\dagger}$ & 0.53 \\
\hline VO2 AT, ml/Kg/minute & $17.6(3.6)$ & $17.8(5.0)$ & $16.7(3.1)$ & $16.0(3.6)$ & 0.70 \\
\hline AT, minutes & $9.0(3.3)$ & $10.1(2.6)$ & $8.3(2.5)$ & $9.7(1.9)^{\S}$ & 0.28 \\
\hline Physical Exercise $(\mathrm{n}=25)^{\|}(\%)$ & $10(40.0)$ & $21(84.0)^{\S}$ & $8(32.0)$ & $20(80.0)^{\S}$ & 0.82 \\
\hline \multicolumn{6}{|c|}{ Exercise, mean \pm sd } \\
\hline frequency, days/week & $5.6(2.0)$ & $6.2(1.7)^{\dagger}$ & $4.7(2.1)$ & $6.1(1.7)^{\dagger}$ & 0.15 \\
\hline duration, minutes/session & 52.7 (24.6) & $53.2(20.8)^{\dagger}$ & $52.2(9.7)$ & $64.2(24.0)^{\ddagger}$ & 0.23 \\
\hline
\end{tabular}

AT, Anaerobic threshold ; HR, heart rate ; VO2AT, Oxygen consumption in AT; VO2p Peak Oxygen Uptake; 6MWT , Six Minute Walking Test; SBP, systolic arterial blood pressure; TP, Training Programme. ${ }^{\dagger} \mathrm{p}<0.05,{ }^{\ddagger} \mathrm{p}<0.01,{ }^{\S} \mathrm{p}<0.001$. p value from TP2 and TP3 comparation through covariance analysis adjusted by basal value for quantitative variables. Logistic regression models were performed with the dependent variable "Practice exercise post TP "Yes/No and with the independent variables: "Practice exercise pre TP" and group (TP2/TP3). ${ }^{\prime \prime}=$ number of patients with pre-TP and post-TP data on exercise.

8-week TP with 3 sessions per week, but their patients were metabolically healthier than those included in our study. In addition, our patients adapted progressively to the exercise intensity, whereas in the study by Boudou and collaborators [15] the exercise intensity was high from the start of the TP. Other studies that used similar TPs [14] [15] where the exercise intensity was increased progressively obtained lower improvement of VO2p than Boudou and collaborators [1]. No significant differences were observed in physical fitness and exercising between patients performing 2 or 3 sessions per week. This suggests that the final result was not influenced by the number of sessions per week. No differences were observed in AT delay between the TP2 and TP3, although a significant delay was observed in TP3 (1.4 min, $\mathrm{p}=0.0008)$, and a trend was observed in TP2 (1.1 min, $\mathrm{p}=$ 0.058). The small sample size and the data variability may explain this lack of significance in the TP2. Patients with 2 sessions per week (TP2), with a total of 16 sessions (the minimum reported in the literature) showed improved physical fitness and exercising. Ligtenberg et al. [17] also observed an improved physical fitness and exercising with a 6 week TP with 3 sessions per week (a total of 18 sessions) but it was performed only on diabetic patients from hospital setting.

We only observed a significant decrease in systolic arterial blood pressure and diastolic arterial blood pressure with the TP, although the analytical parameters and blood pressure may be influenced by the pharmacological adjustments performed during the TP. Other studies performed in primary care setting have also obtained significant decreases in blood pressure, although with longer TP [1] [8]. In a study by Dunstan et al. [15] a decrease in weight and triglyceride was observed. However, their study included individual diet adjustments in addition to a TP.

Our study has several limitations, including the lack of a control group and the lack of randomization in the TP2 and TP3 groups. To minimize the effect of the last point, analyses of subgroups of patients, matched by gender, age and basal data, was performed. The changes in systolic arterial blood pressure and diastolic arterial blood pressure observed may be influenced by changes in pharmacological treatment during the study. However, the main aim of the study was to investigate the effect of the TP on physical fitness. The sample size was sufficiently powered to determine changes in the main endpoint, the 6MWT, but may be insufficient to detect changes in other parameters such as the VO2p.

\section{Conclusion}

In summary, our study shows the effectiveness in physical fitness and exercise of an 8-week TP, with 2 or 3 ses- 
sions per week in patients with a moderate-high risk of CVD. The shortness of the TP facilitates its application to a larger number of patients, enhances compliance, reduces waiting lists and decreases costs. The CPET test is the gold standard for the evaluation of fitness and the prescription of exercise. However, we propose the use of the 6MWT, which is a submaximal test and easy to perform in clinical practice, for the monitoring of the results of this type of TP in older patients, with several co-morbidities and low tolerance of physic effort. This will also facilitate the implementation of TPs in primary care settings, given the simplicity, low risk and low cost of the 6 MWT.

\section{Acknowledgements}

We thank Gloria Saiz Rosell, Mari Carmen Creus and Anna Bosch Mascareta for their collaboration and help with the study.

\section{Statement of Competing Interests}

This manuscript have no conflict of interest.

\section{References}

[1] Eriksson, K.M., Westborg, C.J. and Eliasson, M.C. (2006) A Randomized Trial of Lifestyle Intervention in Primary Healthcare for the Modification of Cardiovascular Risk Factors. Scandinavian Journal of Public Health, 34, 453-461. http://dx.doi.org/10.1080/14034940500489826

[2] Erikssen, G., Liestol, K., Bjornholt, J., Thaulow, E., Sandvik, L. and Erikssen, J. (1998) Changes in Physical Fitness and Changes in Mortality. Lancet, 352, 759-762. http://dx.doi.org/10.1016/S0140-6736(98)02268-5

[3] Varo, J.J., Martínez-González, M.A., De Irala-Estévez, J., Kearney, J., Gibney, M. and Martínez, J.A. (2003) Distribution and Determinants of Sedentary Lifestyles in the European Union. International Journal of Epidemiology, 32, 138146. http://dx.doi.org/10.1093/ije/dyg116

[4] Lindström, J., Ilanne-Parikka, P., Peltonen, M., Aunola, S., Eriksson, J.G., Hemiö, K., et al. (2006) Sustained Reduction in the Incidence of Type 2 Diabetes by Lifestyle Intervention: Follow-Up of the Finnish Diabetes Prevention Study. Lancet, 368, 1673-1679. http://dx.doi.org/10.1016/S0140-6736(06)69701-8

[5] Balducci, S., Zanuso, S., Nicolucci, A., DeFeo, P., Cavallo, S., Cardelli, P., et al. (2010) Effect of an Intensive Exercise Intervention Strategy on Modifiable Cardiovascular Risk Factors in Subjects with Type 2 Diabetes Mellitus. A Randomized Controlled Trial: The Italian Diabetes and Exercise Study (IDES). Archives of Internal Medicine, 170, $1794-$ 1803. http://dx.doi.org/10.1001/archinternmed.2010.380

[6] Boulé, N.G., Kenny, G.P., Haddad, E., Wells, G.A. and Sigal, R.J. (2003) Meta-Analysis of the Effect of Structured Exercise Training on Cardiorespiratory Fitness in Type 2 Diabetes Mellitus. Diabetologia, 46, 1071-1081. http://dx.doi.org/10.1007/s00125-003-1160-2

[7] Snowling, N.J. and Hopkins, W.G. (2006) Effects of Different Modes of Exercise Training on Glucose Control and Risk Factors for Complications in Type 2 Diabetic Patients: A Meta-Analysis. Diabetes Care, 29, 2518-2527. http://dx.doi.org/10.2337/dc06-1317

[8] Isaacs, A.J., Critchley, J.A., Tai, S.S., Buckingham, K., Westley, D., Harridge, S.D., et al. (2007) Exercise Evaluation Randomised Trial (EXERT): A Randomised Trial Comparing GP Referral for Leisure Centre-Based Exercise, Community-Based Walking and Advise Only. Health Technology Assessment, 11, 1-165. http://dx.doi.org/10.3310/hta11100

[9] Eriksson, K.M., Franks, P.W. and Eliasson, M. (2009) A 3-Year Randomized Trial of Lifestyle Intervention for Cardiovascular Risk Reduction in the Primary Care Setting: The Swedish Björknäs Study. PLoS ONE, 4, e5195. http://dx.doi.org/10.1371/journal.pone.0005195

[10] Ariza Copado, C., Gavara Palomar, V., Muñoz Ureña, A., Aguera Mengual, F., Soto Martínez, M. and Lorca Serralta, J.R. (2011) Mejora en el control de los diabéticos tipo 2 tras una intervención conjunta: Educación diabetológica y ejercicio físico. Atención Primaria, 43, 398-406. http://dx.doi.org/10.1016/j.aprim.2010.07.006

[11] Giné-Garriga, M. and Martin-Borrás, C. (2008) Programme in Primary Care Centres to Promote Physical Activity. Pilot Study. Atención Primaria, 40, 374-375. http://dx.doi.org/10.1157/13124135

[12] Arsenault, B.J., Côté, M., Cartier, A., Lemieux, I., Després, J.P., Ross, R., et al. (2009) Effect of Exercise Training on Cardiometabolic Risk Markers among Sedentary, but Metabolically Healthy Overweight or Obese Postmenopausal Women with Elevated Blood Pressure. Atherosclerosis, 207, 530-533. http://dx.doi.org/10.1016/j.atherosclerosis.2009.05.009 
[13] Dunn, A.L., Marcus, B.H., Kampert, J.B., Garcia, M.E., Kohl III, H.W. and Blair, S.N. (1999) Comparison of Lifestyle and Structured Interventions to Increase Physical Activity and Cardiorespiratory Fitness. The Journal of the American Medical Association, 281, 327-334. http://dx.doi.org/10.1001/jama.281.4.327

[14] Maiorana, A., O’Driscoll, G., Goodman, C., Taylor, R. and Green, D. (2002) Combined Aerobic and Resistance Exercise Improves Glycemic Control and Fitness in Type 2 Diabetes. Diabetes Research and Clinical Practice, 56, 115-123. http://dx.doi.org/10.1016/S0168-8227(01)00368-0

[15] Dunstan, D.W., Mori, T.A., Puddey, I.B., Beilin, L.J., Burke, V., Morton, A.R., et al. (1997) The Independent and Combined Effects of Aerobic Exercise and Dietary Fish Intake on Serum Lipids and Glycemic Control in NIDDM. Diabetes Care, 20, 913-921. http://dx.doi.org/10.2337/diacare.20.6.913

[16] Boudou, P., Kerviler, E., Vexiau, P., Fiet, J., Cathelineau, G. and Gautier, J.F. (2000) Effects of a Single Bout of Exercise and Exercise Training on Steroid Levels in Middle-Aged Type 2 Diabetic Men: Relationship to Abdominal Adipose Tissue Distribution and Metabolic Status. Diabetes \& Metab (Paris), 26, 450-457.

[17] Ligtenberg, P.C., Hoekstra, J.B.L., Bol, E., Zonderland, M.L. and Erkelens, D.W. (1997) Effects of Physical Training on Metabolic Control in Elderly Type 2 Diabetes Mellitus Patients. Clinical Science, 93, 127-135.

[18] Expert Panel on Detection, Evaluation, and Treatment of High Blood Cholesterol in Adults (2001) Executive Summary of the Third Report of the National Cholesterol Education Program (NCEP) Expert Panel on Detection, Evaluation, and Treatment of High Blood Cholesterol in Adults (Adults Treatment Panel III). The Journal of the American Medical Association, 285, 2486-2497. http://dx.doi.org/10.1001/jama.285.19.2486

[19] Graham, I., Atar, D., Borch-Johnsen, K., Boysen, G., Burell, G., Cifkova, R., et al. (2007) European Guidelines on Cardiovascular Disease Prevention in Clinical Practice. European Journal of Cardiovascular Prevention \& Rehabilitation, 14, S1-S113. http://dx.doi.org/10.1097/01.hjr.0000277983.23934.c9

[20] Vanhees, L., Geladas, N., Hansen, D., Kouidi, E., Niebauer, J., Reiner, Z., et al. (2012) Importance of Characteristics and Modalities of Physical Activity and Exercise in the Management of Cardiovascular Health in Individuals with Cardiovascular Risk Factors: Recommendations from the EACPR (Part II). European Journal of Preventive Cardiology, 19, 1005-1033. http://dx.doi.org/10.1177/1741826711430926

[21] Manual SEPAR de Procedimientos. Módulo 4. Procedimientos de evaluación de la Función Pulmonar II. Publicaciones Permayer, 2004.

[22] Palange, P., Ward, S.A., Carlsen, K.H., Casaburi, R., Gallagher, C.G., Gosselink, R., et al. (2007) Recommendations on the Use of Exercise Testing in Clinical Practice. European Respiratory Journal, 29, 185-209. http://dx.doi.org/10.1183/09031936.00046906

[23] Wasserman, K., Hansen, J.E., Sue, D.Y., Stringer, W.W. and Whipp, B.J. (2005) Principles of Exercise Testing and Interpretation: Including Pathophysiology and Clinical Applications. Lippincott Williams \& Wilkins, Philadelphia.

[24] Beaver, W.L., Wasserman, K. and Whipp, B.J. (1986) A New Method for Detecting the Anaerobic Threshold by Gas Exchange. Journal of Applied Physiology, 60, 2020-2027.

[25] Wasserman, K., Whipp, B.J., Koyal, S. and Beaver, W.L. (1973) Anaerobic Threshold and Respiratory Gas Exchange during Exercise. Journal of Applied Physiology, 35, 236-243.

[26] Little, P., Dorward, M., Gralton, S., Hammerton, L., Pillinger, J., White, P., Moore, M., et al. (2004) A Randomised Controlled Trial of Three Pragmatic Approaches to Initiate Increased Physical Activity in Sedentary Patients with Risk Factors for Cardiovascular Disease. British Journal of General Practice, 54, 189-195.

[27] Weltman, A., Snead, D., Stein, P., Seip, R., Schurrer, R., Rutt, R., et al. (1990) Reliability and Validity of a Continuous Incremental Treadmill Protocol for the Determination of Lactate Threshold, Fixed Blood Lactate Concentrations, and VO2max. International Journal of Sports Medicine, 11, 26-32. http://dx.doi.org/10.1055/s-2007-1024757

[28] Yoshida, T. (1984) Effect of Exercise Duration during Incremental Exercise on the Determination of Anaerobic Threshold and the Onset of the Blood Lactate Accumulation. European Journal of Applied Physiology and Occupational Physiology, 53, 196-199. http://dx.doi.org/10.1007/BF00776589 


\section{List of Abbreviations}

CVD: Cardiovascular Disease

TP: Training Programme

AT: Anaerobic Threshold

CPET: Cardiopulmonary Exercise Testing

6MWT: Six Minute Walking Test

VO2p: Peak Oxygen Uptake 